\begin{tabular}{|c|c|c|}
\hline $4 . E g$ & $\begin{array}{c}\text { European Association for the } \\
\text { Development of Renewable Energies, Environment } \\
\text { and Power Quality (EA4EPQ) }\end{array}$ & $\begin{array}{c}\text { International Conference on Renewable Energies and Power Quality } \\
\text { (ICREPQ'12) }\end{array}$ \\
Santiago de Compostela (Spain), 28th to 30th March, 2012
\end{tabular}

\title{
Power Quality in Renewable Energy Systems - Challenges and Opportunities
}

\author{
Jian Sun \\ Department of Electrical, Computer, and Systems Engineering \\ Rensselaer Polytechnic Institute \\ 110 8th Street, Troy, NY 12180, USA \\ Telephone: (518) 276-8297; email: jsun@rpi.edu
}

\begin{abstract}
Power quality in traditional power systems is concerned with voltage problems caused by abnormal operation or nonlinear load behavior. The integration of renewable energy into the power grid introduces new power quality problems. In addition to the intermittency of renewable production, which leads to more frequent voltage fluctuation, and the switching in the power electronics interfaces, which injects high-frequency harmonics into the system, harmonic resonance and other dynamics power quality problems become more prevalent due to the complex and fast dynamics and control of power electronics interfaces. This paper reviews the sources of harmonic resonance involving renewable energy and presents a systematic method to identify and characterize such dynamic power quality problems. A distributed generation test-bed is also presented as an experimental platform for the study of system harmonic resonance and other control-related power quality and stability problems. Measurement results involving single-phase solar and three-phase wind inverters are presented to validate the analysis method and to demonstrate possible mitigation techniques. The need for advanced autonomous control that enhances system stability and mitigates system harmonic resonance are discussed. Issues related to electromagnetic interference (EMI) generated by renewable energy sources and the need to develop EMI compliance requirements for such sources are also discussed.
\end{abstract}

\section{Key Words}

Harmonic Resonance, Impedance Analysis, Dynamic Power Quality, Electromagnetic Interference

\section{Introduction}

Traditional power systems are dominated by slowresponding electromechanical and electromagnetic energy conversion devices, such as generators, motors, and transformers (as well as passive loads.) The physical design of a synchronous generator guarantees the generation of sinusoidal voltages (see Fig. 1a), such that only the speed of the generator needs to be regulated in order to maintain a constant frequency. Control of the generator speed is also important for achieving synchronous operation (angular stability) of multiple generators. Synchronous generators have a tendency to stay in synchronism with each other once they are synchronized, which helps to simplify system control. Additionally, the inductive nature of long transmission lines allows voltage control to be separated
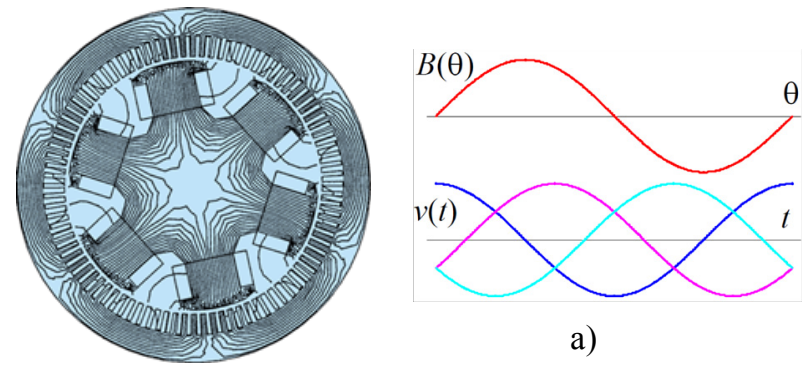

a)

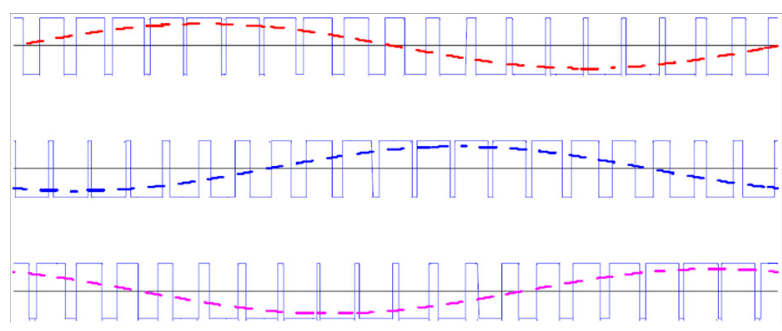

b)

Fig. 1. a) A synchronous generator produce sinusoidal voltages by virtue of sinusoidally distributed magnetic field or windings; b) Synthesis of sinusoidal voltages by pulse-width modulation for integration of renewable energy into the grid.

from frequency control, and to be achieved through reactive power regulation. Although voltage control can be faster than frequency control, the voltage control bandwidth typically still doesn't exceed a few Hz. As the result, the dynamics of a traditional power system are concentrated below the system fundamental frequency [1].

Large-scale integration of renewable energy is fundamentally changing the characteristics of the power grid. Counteracting the intermittence of renewable sources requires a combination of a variety of technologies, including geographical distribution with strong and flexible transmission lines incorporating FACTS and HVDC, energy storage of various scales and at different levels, as well as demand response and dynamic load management. Power electronics is a critical enabler; in particular, almost all renewable sources require a power electronics interface for integration into the grid. In addition to traditional performance metrics such as cost, efficiency, and reliability, new control functions must be developed to support a smart grid infrastructure. 
One particular issue related to the ubiquitous use of power electronics in future power systems is the extended control bandwidth of power electronics circuits and the resulting fast and complex system dynamics beyond the grid fundamental frequency. Unlike a synchronous generator, a power electronics circuit synthesizes sinusoidal ac voltages from dc sources by means of pulse-width modulation (PWM), as illustrated in Fig. 1b). The PWM (switching) frequency depends on the power level and can range from a few hundred $\mathrm{Hz}$ to hundreds of $\mathrm{kHz}$. Synthesizing a $50 / 60 \mathrm{~Hz}$ sinusoidal voltage/current also requires a control bandwidth at least 10 times higher than the line fundamental frequency. Fig. 2 compares the typical speed of control functions between a traditional synchronous generator and a large wind turbine.

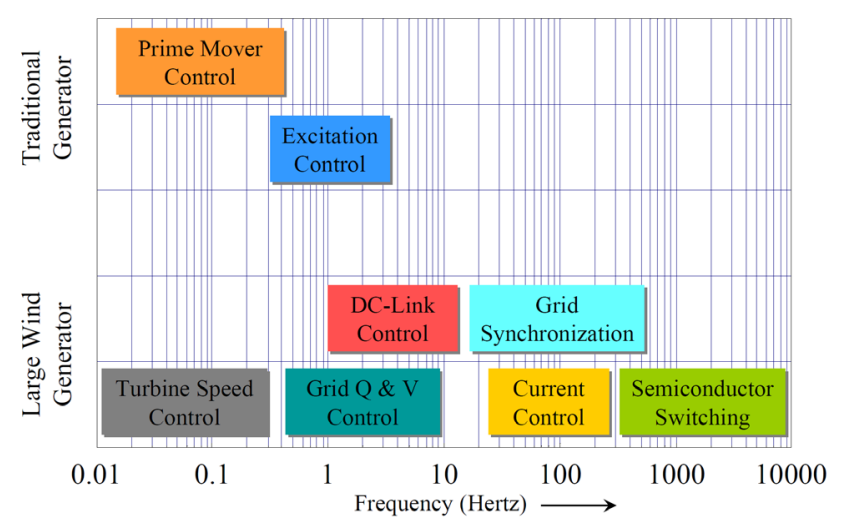

Fig. 2. Comparison of the typical speed of control functions for a traditional generator and a large wind turbine.

Power quality in traditional power systems is usually treated separately from system dynamic behavior and stability [2]. Harmonics, for example, are considered a steady-state problem caused by nonlinear effects which can be modeled as harmonic current sources. Stability analysis, on the other hand, is concerned with system dynamics below the fundamental frequency. Such a separation between system stability and power quality disappears in renewable energy systems due to the wide control bandwidth of power electronics interfaces. Instability or lack of stability margin may occur both below and above the line fundamental frequency and, in the latter case, can manifests itself into a harmonic distortion problem. A typical example is a grid-connected inverter for solar or wind energy integration. The inverter output impedance is usually capacitive within its current control bandwidth, which can range from a few hundred $\mathrm{Hz}$ to several $\mathrm{kHz}$ depending on the inverter power rating and switching frequency. Such capacitive output impedance can form a resonance with the grid impedance, which is usually inductive, and create severe harmonic distortion at the grid interconnect point.

The blending of stability and power quality problems in renewable energy systems brings new challenges as well as opportunities. Existing power system theories are built upon phasor-based models which cannot capture fast dynamics above the line frequency. To overcome this limitation, an impedance-based method has been developed as an integrated framework for the study of both system stability and power quality. A distributed generation (DG) test-bed has also been developed as an experimental platform to support theoretical study and to demonstrate advanced system control techniques. The testbed provides a controlled distribution grid environment that can be programmed to simulate various grid configurations and operation conditions.

This paper reviews the sources of harmonic resonance involving renewable energy sources, and presents an overview of the impedance-based system analysis techniques as well as the DG test-bed. Measurement results involving single-phase and three-phase inverters are also presented to validate the analysis method and to demonstrate possible mitigation techniques. The needs for advanced autonomous control that enhances system stability and mitigates system harmonic resonance are presented. Issues related to electromagnetic interference (EMI) generated by renewable energy sources and the need to develop EMI compliance requirements for such sources are also discussed.

\section{Harmonic Resonance and Stability}

Sustained current and/or voltage resonance is a common stability problem which often occurs in systems involving power electronic sources and loads. Ref. [3] discussed input harmonic current distortion in single-phase power factor correction (PFC) converters due to undamped input EMI filter interacting with the source output impedance. Ref. [4] presented resonance-related instability of dc bus voltage in motor drive systems with a line-frequency rectifier as the front-end. A common source of harmonic resonance is the constant-power behavior of power electronic loads. As discussed in [5], a constant-power load exhibits negative incremental resistance which destabilizes the system. Proper damping of input and output EMI filters is important and can effectively eliminate such resonance [6].

Renewable energy sources are mostly integrated into the grid through PWM inverters that are controlled to behave as current sources, that is, to inject into grid active currents that depend on the amount of energy available from the renewable sources. Injection of reactive currents start to be required as a way to improve system voltage regulation and stability. In particular, wind inverters are required now to stay connected with the grid under low or even zero grid voltage conditions, as well as to regulate the grid voltage at the point of common coupling (PCC). Harmonic resonance has been found to be an increasingly common problem at the interface between such inverters and the grid. As mentioned in the previous section, the root cause of such resonance is that a PWM inverter exhibits capacitive output impedance within its current control bandwidth.

To demonstrate such harmonic resonance problems, Fig. 3 shows measurements of a single-phase small wind inverter output voltage and current when connected to a grid through variable impedance. The inverter is rated at $6 \mathrm{~kW}$, and the grid is established by the grid simulator which 

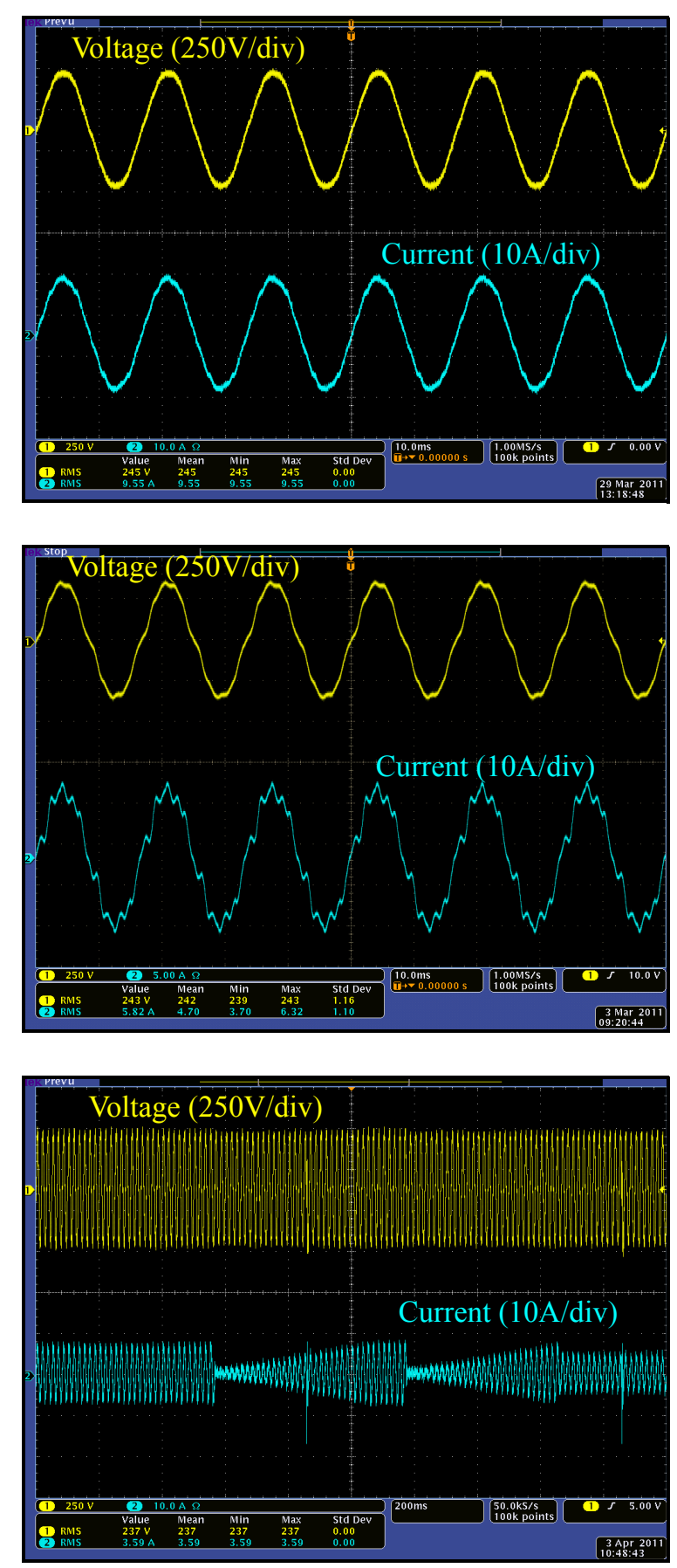

Fig. 3. Operation of a single-phase wind inverter with different grid impedance.

allows the effective grid impedance seen by the inverter to be varied, as described in Section 5. Three different measurements are shown:

1) Normal Operation: The test-bed distribution network was connected to the utility grid with no additional impedance. The impedance seen by the inverter was therefore very low. Both the grid voltage and the inverter output current were almost sinusoidal in this case and there was no operational issue.

2) Distorted Operation: The test-bed was configured to effectively insert $6 \mathrm{mH}$ inductance between the inverter and the utility grid. The high grid impedance created a harmonic resonance with the inverter, leading to highly distorted inverter output current and voltage.
3) Unstable Operation: The inserted inductance is further increased to $12.5 \mathrm{mH}$. The inverter could not stay connected to the grid reliably in this case and was tripped off periodically. Both the grid voltage and the inverter output current were highly distorted during the time when the inverter was still connected to the grid.

The harmonic distortion demonstrated in these measurements is the result of dynamic interactions between the inverter and the grid, not by nonlinear characteristics of any loads, which are the typical sources of harmonics in traditional power systems, nor is it due to the PWM switching of the semiconductor devices in the inverter, as the inverter switching frequency is much higher. The problem is similar to the harmonic resonance between a rectifier and the source [3], as well as the more general source-load interaction problems in power electronics systems. The problem is essentially due to the influence of grid impedance on the dynamics and stability of the inverter control loops.

The existing approach to the analysis of grid-connected inverter stability in the presence of grid impedance is to model the inverter and the grid together as a whole system [7]. This approach requires full knowledge of both the inverter and the grid, and is inconvenient to use for either the inverter designer, who typically doesn't know exactly the grid characteristics the inverter has to work with, or the system integrator, who usually doesn't have access to the internal design of the inverter. The impedance-based approach [8] presented in the next section solves this problem by allowing the stability of the inverter in the presence of grid impedance to be assessed by simply using the inverter output impedance and grid impedance.

\section{Impedance-Based Analysis Method}

The essence of the impedance-based system stability criterion is to partition the system under study into a source and a load subsystem. The source subsystem is modeled by its Thevenin equivalent circuit consisting of an ideal voltage source $\left(V_{s}\right)$ in series with an output impedance $\left(Z_{S}\right)$, while the load subsystem is modeled by its input impedance $\left(Z_{l}\right)$, see Fig. 4. Since almost all power electronic circuits are nonlinear, this linear representation is only valid for small-signal analysis.

With the assumed small-signal model, the current $I$ flowing from the source to the load is

$$
I(s)=\frac{V_{s}(s)}{Z_{l}(s)} \cdot \frac{1}{1+Z_{s}(s) / Z_{l}(s)}
$$

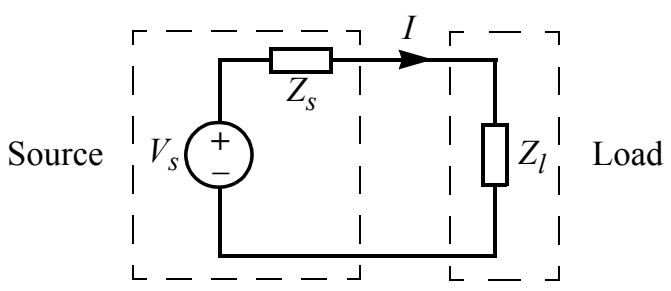

Fig. 4. Small-signal representation of a voltage source with load. 
For system stability analysis, it can be assumed that the source voltage is stable when unloaded and the load current is stable when powered from an ideal source. In that case, both $V_{s}(s)$ and $1 / Z_{l}(s)$ are stable, such that stability of the current depends on the stability of the second term on the right-hand side of (1):

$$
H(s)=\frac{1}{1+Z_{s}(s) / Z_{l}(s)}
$$

The impedance-based stability criterion is based on the observation that $H(s)$ resembles the close-loop transfer function of a negative feedback control system, where the forward gain is unity and the feedback gain is $Z_{s}(s) / Z_{l}(s)$; that is, the ratio of the source output impedance to the load input impedance. By linear control theory, $H(s)$ is stable if and only if $Z_{s}(s) / Z_{l}(s)$ satisfies the Nyquist stability criterion [9].

\section{A. Current-Source System Stability}

When applying the impedance-based stability criterion presented above, it is important to recognize that the source is assumed to be a voltage source that is stable when unloaded. Since most practical sources are voltage sources and are stable when unloaded, this assumption tends to be forgotten. However, grid-connected inverters are usually controlled in the current-injection mode and don't behave like a voltage source. Hence their stability cannot be analyzed by the existing impedance-based method.

To develop an impedance-based stability criterion for current-source systems, we start from a small-signal model similar to that used in the voltage-source case. However, instead of using a Thevenin equivalent circuit, we represent the current source by a Norton equivalent circuit in the form of a current source $\left(I_{\mathrm{s}}\right)$ in parallel with an output admittance $\left(Y_{\mathrm{S}}\right)$. The load is represented by its input admittance, $Y_{l}$, see Fig. 5. With this small-signal representation, the voltage across the load is

$$
V(s)=\frac{I_{s}(s)}{Y_{l}(s)} \cdot \frac{1}{1+Y_{s}(s) / Y_{l}(s)} .
$$

Similar to the voltage-source case, the current source can be assumed stable when unloaded (that is, when the load is a short-circuit such that its admittance is infinite), and the load is stable when powered from an ideal current source. Under these assumptions, both $I_{s}(s)$ and $1 / Y_{l}(s)$ are stable, such that the stability of $V(s)$ depends on the stability of the second term on the right-hand side of (3). Note again that this term resembles the close-loop transfer

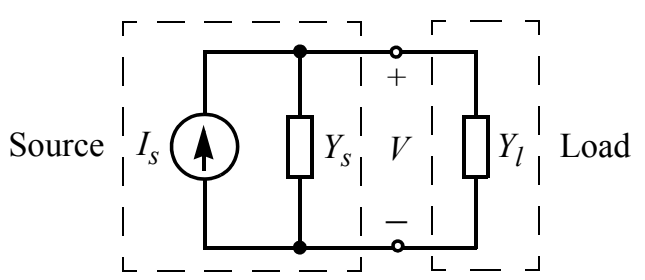

Fig. 5. Small-signal representation of a current source with load. function of a negative feedback control system where the forward gain is unity and the feedback gain is $Y_{s}(s) / Y_{l}(s)$. Therefore, a current-source system is stable if

1) The current source itself is stable when unloaded, that is, when the load is a short-circuit;

2) The load is stable when supplied by an ideal current source; and

3) The ratio of the source output admittance to the load input admittance satisfies the Nyquist criterion.

Note that admittances instead of impedances are used in (3) in order to highlight its duality to (2). The analysis can also be carried out based on the source output impedance $\left(Z_{S}\right)$ and load input impedance $\left(Z_{l}\right)$, in which case (3) becomes

$$
V(s)=I_{s}(s) Z_{l}(s) \cdot \frac{1}{1+Z_{l}(s) / Z_{s}(s)}
$$

and stability of the interconnected system requires the ratio of the load input impedance to the source output impedance meet the Nyquist stability criterion.

Comparing (4) to (2), one can see that stability requirements for current-source systems are opposite to that for voltage-source systems: A current source should have high (ideally infinite) output impedance, while a voltage source should have low (ideally zero) output impedance in order to ensure stable operation with a wide range of loads; and a current-source system is more stable when the load impedance is low, while a voltage-source system is more stable when the load impedance is high.

Given that Thevenin and Norton equivalent circuits can be used exchangeably in circuit analysis, one might ask why it is necessary to make a distinction between currentsource and voltage-source systems. Further, one may argue that a current-source system could also be represented by the Thevenin equivalent circuit, such that, based on (2), stability of the system would require the ratio of the source output impedance to the load input impedance, not the inverse as appeared in (4), to satisfy the Nyquist stability criterion. These questions are addressed in [8].

\section{B. Grid-Connected Inverter Stability}

The most common grid model for system stability analysis is an ideal voltage source in series with a grid impedance, $Z_{g}$, typically consisting of an inductor in series with a resistor. With the grid-connected inverter modeled as a current source in parallel with an output impedance, the overall inverter-grid system can be represented by the small-signal equivalent circuit shown in Fig. 6. This can be viewed as a hybrid system consisting of both a voltage source and a current source. Consistent with the previous assumptions and common practice, the grid voltage can be assumed to be stable without the inverter, and the inverter to be stable when the grid impedance is zero. Based on the equivalent circuit, the inverter output current is

$$
I(s)=\frac{I_{c}(s) Z_{0}(s)}{Z_{0}(s)+Z_{g}(s)}-\frac{V_{g}(s)}{Z_{0}(s)+Z_{g}(s)}
$$




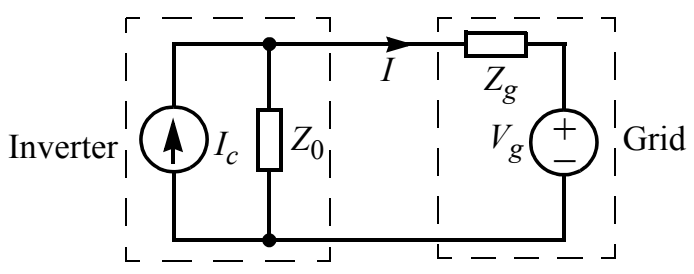

Fig. 6. Small-signal representation of an inverter-grid system.

which can be rearranged to

$$
I(s)=\left[I_{c}(s)-\frac{V_{g}(s)}{Z_{0}(s)}\right] \cdot \frac{1}{1+Z_{g}(s) / Z_{0}(s)} .
$$

Based on (6) and the assumptions stated before, a gridconnected inverter will operate stably if the ratio of the grid impedance to the inverter output impedance, $Z_{g}(s) / Z_{0}(s)$, satisfies the Nyquist criterion. Stability margin of the system can also be measured from the Nyquist plot of $Z_{g}(s) / Z_{0}(s)$. Note that one arrives at the same conclusion if the system is treated as a voltagesource system powered by the grid.

\section{Impedance Modeling}

Proper impedance models are required for the application of the impedance-based system stability criterion presented in the previous section. The grid can usually be modeled by an inductor or an inductor in series with a resistor. Such simple RL model, however, will no longer be valid in the future when a significant portion of system power is supplied from renewable sources through inverters. More specifically, the grid impedance would be dominated more by inverters that integrate renewable sources than traditional generators and transmission/distribution networks. Since all inverters are nonlinear, smallsignal analysis is necessary in order to develop their output impedance models for system stability analysis.

A fundamental difficulty in developing small-signal impedance models for grid-connected inverters and other power electronics devices is that the system operates along a sinusoidal trajectory and lacks a constant operation point about which the system model can be linearized by conventional small-signal analysis methods. Traditional power system theories overcome this difficulty by representing the system by voltage and current phasors [1]. Since both the amplitude and phase of a phasor are constant when the system is in steady-state, the nonlinear phasor model can be linearized by conventional smallsignal methods to develop a linear system model for stability analysis and control design. However, such phasor-based models are only valid below the line fundamental frequency and, hence, cannot be used for the analysis of harmonic resonance and other high-frequency phenomena. Additionally, a phasor model cannot be used to determine the input and output impedance of a converter [9], hence is not compatible with the impedance-based system analysis approach.

A balanced three-phase system can be transformed into a rotating dq reference frame, in which balanced three-phase sinusoidal voltages and currents become dc quantities when the rotating speed of the reference frame is synchronized to the fundamental frequency. This technique is widely used in motor drive control design and has been applied to study the stability of three-phase PWM converters. One limitation of this method is that the transformed model is coupled in the dq-coordinate system, such that the generalized Nyquist criterion has to be applied [10], which is more complex. The method also suffers from several other limitations [9]:

1) The method cannot be applied to single-phase systems, or three-phase systems with significant single-phase loads. A unbalanced three-phase system can be transformed, but the 0 -axis variables will be non-zero and change periodically with time, making it impossible to linearize the model.

2) In many cases, there is no clear physical interpretation for the impedances in the $\mathrm{d}$ - and q-axis as well as the coupling between them. Experimental measurement of such impedances is also difficult because of the requirement for special equipment and instruments.

3) The model in the dq coordinate system remains timevarying if any of the three-phase variables contains more than one sinusoidal component. Hence the method cannot be applied to study the effects of harmonics on system stability.

To overcome the limitations of the phasor and the dqtransformation method, we have developed a new smallsignal analysis method called harmonic linearization. Harmonic linearization is a technique that develops a small-signal linear model for a nonlinear system along a periodic, time-varying operation trajectory. The operation trajectory may comprise a single or multiple sinusoidal (harmonic) components. The method is conceptually simple, involving superimposing harmonic perturbation to the excitation of the system, determining the resulting responses of variables of interests, and extracting the corresponding components at the perturbation frequency by invoking the harmonic balance principle and smallsignal approximation, that is, by assuming that the harmonic perturbation is sufficiently small. The method was first developed to model the input impedance of linecommutated converters [11, 12], and was recently extended to model PWM inverters and converters for highvoltage dc (HVDC) applications [13-15].

In addition to its general applicability, the harmonic linearization method has several other advantages, particularly compared to modeling in the dq-coordinate system:

1) The method effectively decomposes a three-phase system into a positive-sequence and a negativesequence subsystem ${ }^{\dagger}$ without cross coupling between them. Since the sequence transformation is linear and time-invariant, a system is stable if and only if it is stable in all sequence domains.

2) The steady-state operation trajectory can include any number of harmonics, as demonstrated in [12] for a

$\dagger$. As well as a zero-sequence subsystem if unbalance conditions exist and a zero-sequence current can flow. 
single-phase diode rectifier.

3) The method can handle both balanced and unbalanced systems, including single-phase systems.

4) The resulting impedance models can be valid both above and below the ac fundamental frequency, depending only on the characteristics of the converter.

5) The calculated impedance has clear physical interpretations and can be directly measured.

As an example, a PWM inverter employing direct phase current control can be modeled by the following impedances, where $H_{i}(s)$ is the transfer function of the phase current compensator, $L$ is the output inductance, $V_{\mathrm{dc}}$ is the dc bus voltage, $T_{\mathrm{PLL}}$ is the loop gain of the phase-locked loop (PLL) that is used to synchronize with the grid, and $I_{1}$ and $\phi_{i 1}$ are the amplitude and phase angle of the output current, respectively:

1) Positive-Sequence Output Impedance:

$$
Z_{p}(s)=\frac{H_{i}(s) V_{\mathrm{dc}} / 2+s L}{1-\frac{1}{2} T_{\mathrm{PLL}}\left(s-j 2 \pi f_{1}\right)\left[H_{i}(s) \frac{V_{\mathrm{dc}} I_{1}}{2} e^{j \phi_{i 1}}\right]}
$$

2) Negative-Sequence Output Impedance:

$$
Z_{n}(s)=\frac{H_{i}(s) V_{\mathrm{dc}} / 2+s L}{1-\frac{1}{2} T_{\mathrm{PLL}}\left(s+j 2 \pi f_{1}\right)\left[H_{i}(s) \frac{V_{\mathrm{dc}}}{V_{1}} \frac{I_{1}}{2} e^{-j \phi_{i 1}}\right]}
$$

\section{Distributed Generation Test-Bed}

To support the research on renewable energy system power quality and stability using the impedance-based approach, a controlled grid environment, in which the grid impedance can be programmed to emulate different grid conditions, is needed. We have developed such an experimental platform using financial support received from New York State Foundation for Science, Technology and Innovation (NYSTAR). Fig. 7 and 8 show the overall layout and pictures of several key components of this distributed generation (DG) system test-bed.

At the core of our DG test-bed is a power electronics-based grid simulator which can be programmed to provide a local distribution grid with variable voltage, frequency, harmonic contents, as well as impedance characteristics, making it possible to experimentally investigate renewable energy grid integration issues under different grid conditions. The grid simulator is developed using two threephase voltage-source converters connected back-to-back between the test-bed and the utility grid, see Fig. 8. Its bidirectional power capability allows power to be supplied from the grid to the test-bed or moved from the test-bed to the grid. The converter connecting to the test-bed is made of three voltage-source converters operating in parallel, with $60 \mathrm{~kW}$ total power capacity. The three parallel converters are controlled by interleaved pulse-width modulation to minimize harmonic filtering requirements as well as to enable high switching frequency required for wide control bandwidth. Each converter module is
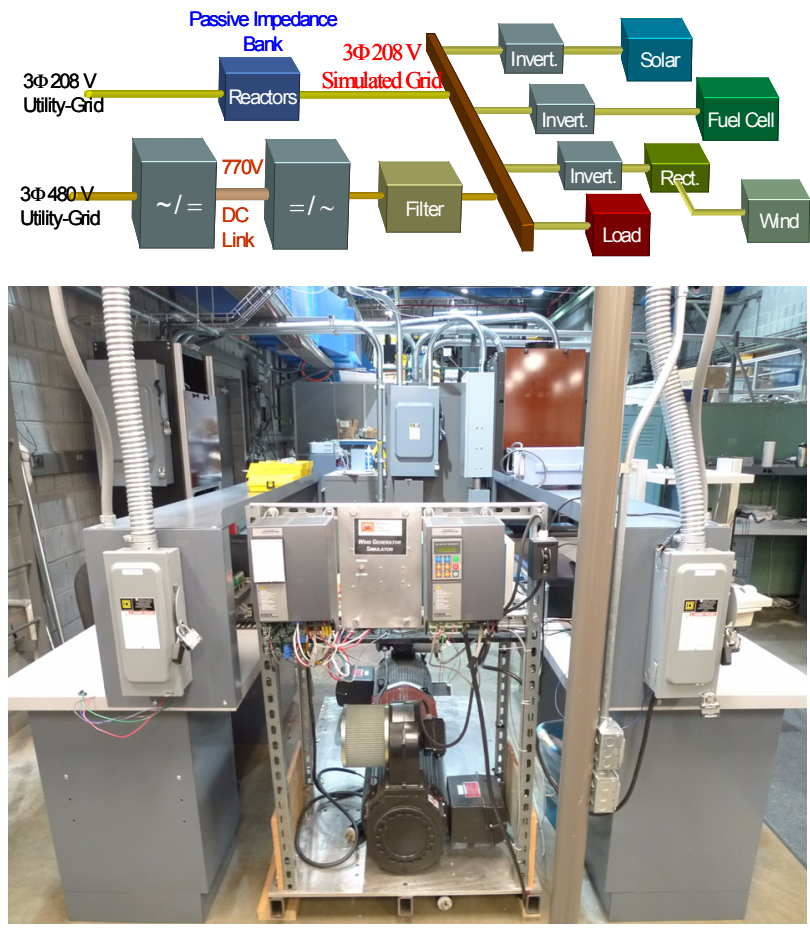

Fig. 7. Overall layout of the distributed-generation system testbed (above) and a picture showing key components of the system (bottom).

controlled by an FPGA, and a digital signal processor (DSP) coordinates the operation of all three modules. Variable voltage, frequency, harmonics, and impedance is achieved through programming of the converter control. The grid simulator output is distributed through the testbed as a programmable local grid.

Active control can affect the grid simulator output impedance within its control bandwidth, which is limited by the switching frequency. To provide additional programmability for the impedance, a passive impedance bank consisting of variable inductors and capacitors has also been built. The impedance bank can connect the testbed distribution network to the utility grid through its variable impedance. It can also operate in parallel with the active grid simulator to expand the range of programmability of the grid impedance. The passive impedance bank was designed to handle the full power of the test-bed, which is rated for $75 \mathrm{~kW}$ in three-phase configuration and $25 \mathrm{~kW}$ in single-phase configuration.

In addition to the grid simulator and passive impedance bank, the test-bed also includes the following major equipment and components:

1) Three single-phase solar inverters each rated for $3 \mathrm{~kW}$ and having two separate MPPT input channels. Each solar inverter can be supplied from actual solar panels or from a solar panel simulator which was developed by using a programmable power supply in conjunction with a DSP control board. The DSP measures the solar inverter input current and use a representative solar panel model to determine the programmable source output voltage.

2) Twenty $200 \mathrm{~W}$ solar panels each equipped with a microinverter that is capable of directly interfacing with the test- 


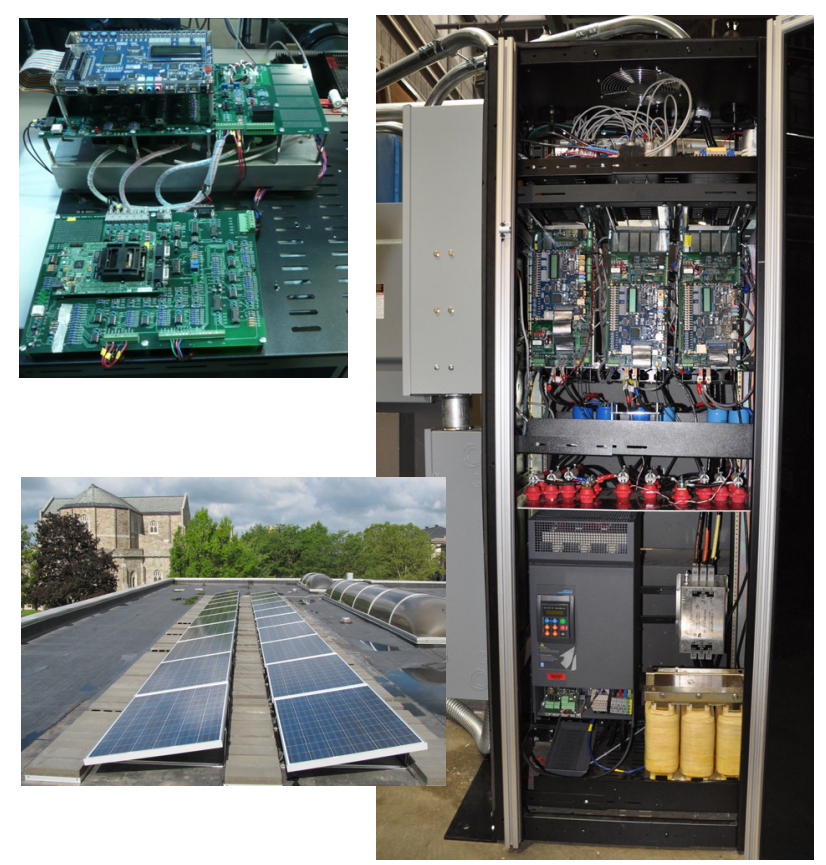

Fig. 8. Active grid simulator, its voltage-source converter building block, and solar panels with micro-inverter.

bed. The solar panels are installed on the roof of the lab (see Fig. 8) and are fed directly into the test-bed.

3) A $15 \mathrm{~kW}$ wind power generation system based on a motor-generator set. The motor is controlled by a variable-speed drive and can be programmed to emulate the operation of a wind turbine under variable wind conditions. The generator output is rectified using an actively controlled rectifier and then connected to the test-bed distribution network through a three-phase PWM inverter. Both the motor and the generator are custom-designed permanent-magnet machines with low rated speeds suitable for direct-drive wind applications.

4) A $7 \mathrm{~kW}$ fuel-cell-based DG unit consisting of a singlephase grid-parallel inverter and a fuel cell simulator. The fuel cell simulator is developed using similar hardware and software as used by the solar panel simulator.

5) A local distribution network with necessary protection devices and instrumentation to allow each of the DG units and various loads to be connected to the grid simulator for system testing.

6) Electronic ac loads that can be programmed to emulate different load characteristics (resistive, capacitive, constant current, constant power, etc.) for loading of the gird simulator and different DG units.

7) A battery-based energy storage system is currently under development and will be added to the test-bed in the future.

The grid simulator can be configured to simulate different grid conditions and system architectures, including micro grids. Currently we are adding an electric car as both load and energy storage device, and will use it to study the effects of electrical transportation on future distribution system, particularly in conjunction with renewable energy. The test-bed is also available for collaboration with researchers from other institutions.

\section{Analysis and Mitigation of Harmonic Reso- nance}

This section presents sample measurement results involving single-phase and three-phase inverters and the test-bed to demonstrate the harmonic resonance problem and the application of impedance-based analysis method.

\section{A. Single-Phase Inverter}

A $3 \mathrm{~kW}$ commercial single-phase solar inverter was measured on the test-bed. Two sets of test results are presented here. In the first case, the inverter was connected to the grid directly, and the inverter output current and voltage waveforms are shown in Fig. 9a), which indicate stable operation. In the second case, an additional inductor $L_{p}=12.8 \mathrm{mH}$ was inserted to purposely increase the grid impedance. The

a)

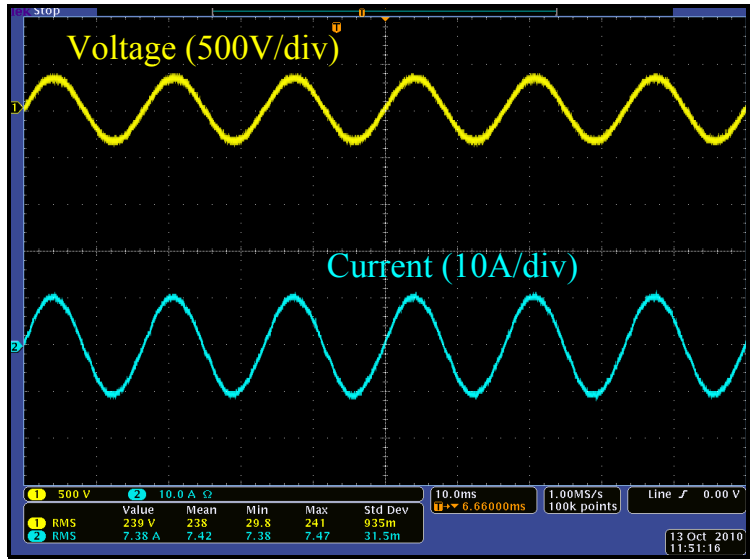

b)
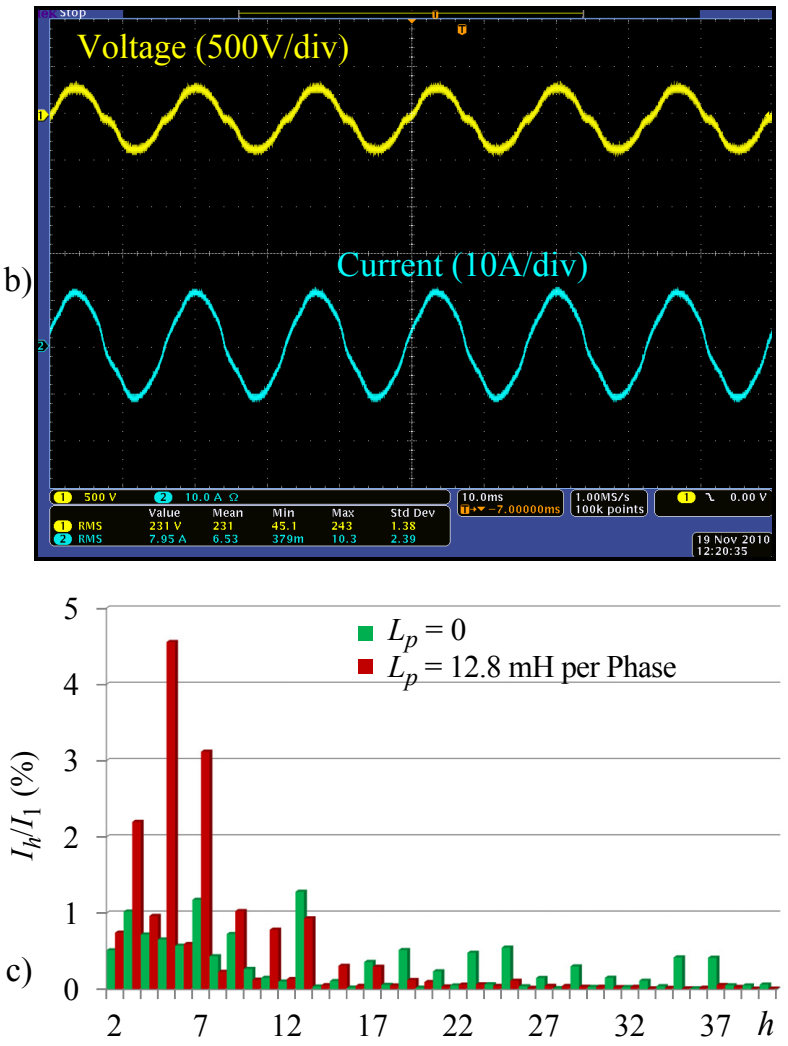

Fig. 9. Solar inverter responses under different grid conditions. a) Passive impedance bank short-circuited; b) Passive impedance bank configured to insert $12.8 \mathrm{mH}$ inductance; c) grid current harmonics for each of the cases. 
measured inverter output current and voltage waveforms are presented in Fig. 9b). The grid current spectrum, shown in Fig. 9c), indicates significant $5^{\text {th }}$ and $7^{\text {th }}$ harmonics. The inverter output power was $1700 \mathrm{~W}$ in both cases.

To apply the impedance-based stability criterion presented in the previous section, the output impedance of the inverter and the grid impedance was measured by using a frequency analyzer for each test condition. Fig. 10 shows the measured frequency responses of the inverter output impedance and the grid impedance for the two cases tested. Without the additional inductor $L_{p}$, the grid impedance intersects with the inverter output impedance at three different frequencies (3 $\mathrm{kHz}, 8 \mathrm{kHz}$, and $25 \mathrm{kHz}$ ) where the phase difference is $115^{\circ}$, $-20^{\circ}$, and $110^{\circ}$, respectively, indicating sufficient phase margin in system stability at all points. With the additional $12.8 \mathrm{mH}$ inductor, the grid impedance intersects with the inverter output impedance only once at about $420 \mathrm{~Hz}$, where the phase difference is about $160^{\circ}$. This intersection frequency correlates closely with the dominant harmonic frequencies in the measured inverter output current and voltage, indicating that the harmonics are caused by a marginally stable resonance between the inverter and the grid impedance at this frequency.
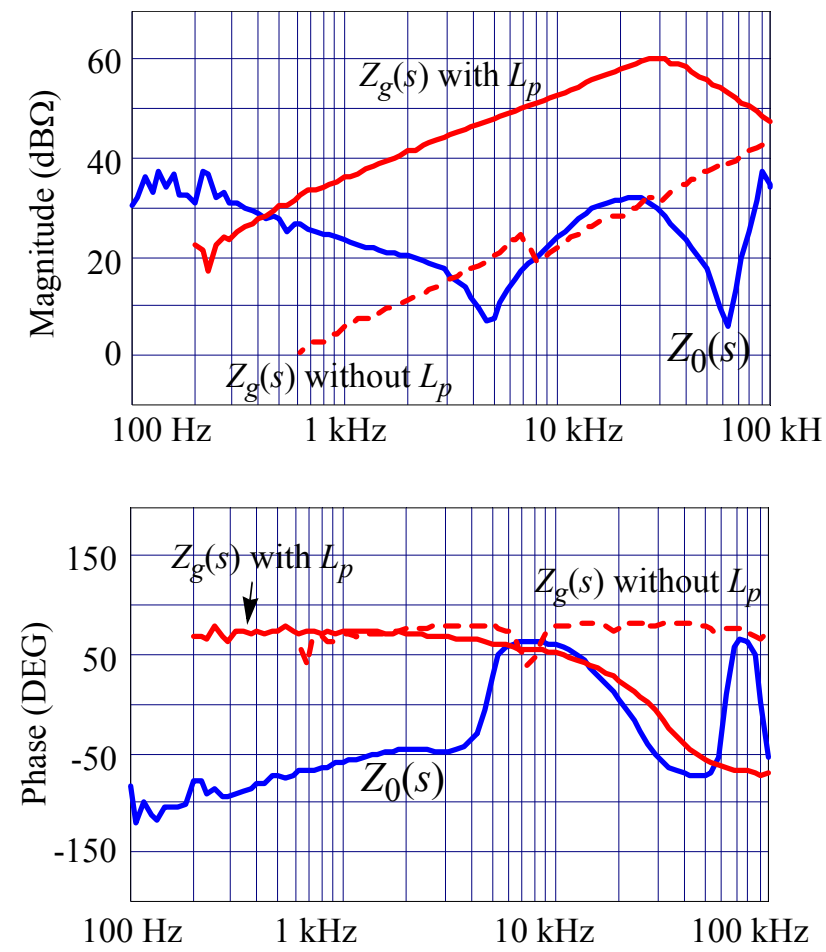

Fig. 10. Frequency responses of the single-phase solar inverter output and the grid impedance.

\section{B. Three-Phase Inverter}

A three-phase PMW inverter was implemented using the same voltage-source converter that was used to build the active grid simulator in the test-bed. The inverter uses a PLL to synchronize with the grid, and a PI regulator to control the phase current. An additional high-frequency pole was used in the current compensator to reduce high-frequency ripple in the current. The output impedances of the inverter are described by (7) and (8) given in Section 4.
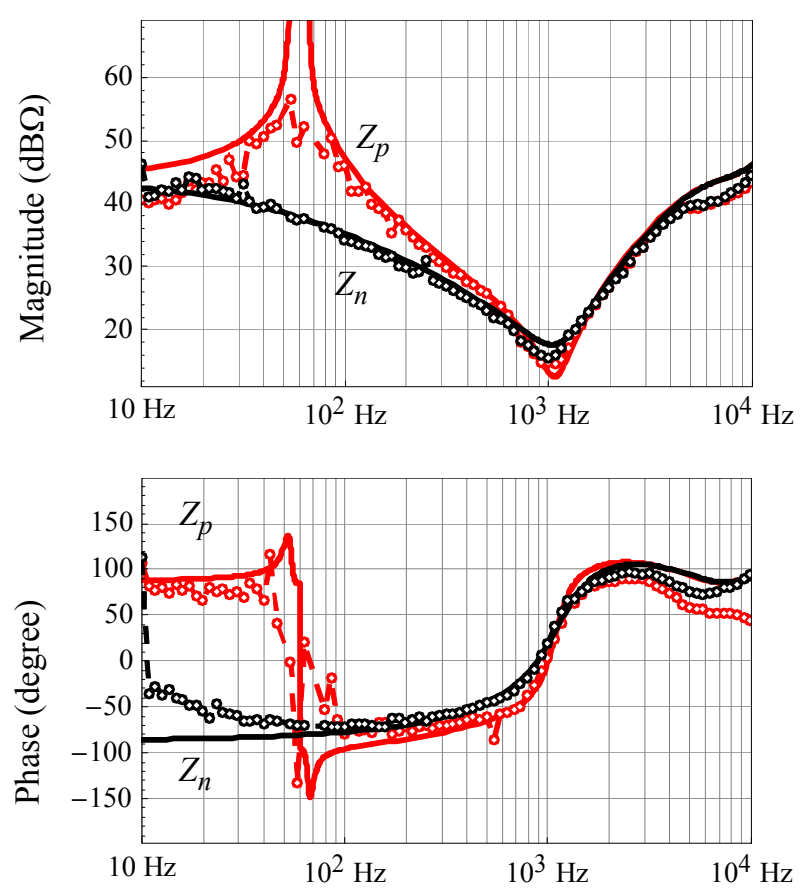

Fig. 11. Positive- and negative-sequence output impedance responses of a three-phase inverter. Solid lines: model prediction; Dash-dot: experimental measurements.

The control was implemented in a digital signal processor TMS320F28335. The digital control introduces a delay in the current control loop, which affects the output impedance. Given the sampling time, $T_{s}$, and computation delay, $T_{d}$, the effects can be included by adding a transfer function

$$
e^{-s T_{d}\left(\frac{1-e^{-s T_{s}}}{s T_{s}}\right)}
$$

to the PWM output. In experiments, the total time delay was measured to be $78 \mu \mathrm{s}$. The frequency responses of the inverter output impedance are predicted using the impedance models and are compared in Fig. 11 with the actual measurements of the inverter output impedance.

The inverter was measured in the test-bed by configuring the passive grid simulator to effectively provide $4.9 \mathrm{mH}$ grid impedance. To demonstrate the effects of inverter control on the stability of the inverter-grid system, we tested the inverter with two different bandwidths of the PLL. In the first case, the PLL bandwidth was set at $100 \mathrm{~Hz}$, and the corresponding current responses of the inverter are shown in Fig. 12a). Large distortion due to harmonic resonance between the inverter and the grid can be seen. Fig. 12c) shows the spectrum of the inverter output current and the Nyquist plot of the ratio of the grid impedance to the inverter output impedance. The positive impedance ratio shows that the system is stable but with only less than $20^{\circ}$ phase margin. The small phase margin leads under-damped resonance and explains the observed strong $3^{\text {rd }}$ and $4^{\text {th }}$ harmonics.

Fig. 12b) shows the measured current waveforms when the PLL bandwidth is reduced to $10 \mathrm{~Hz}$ PLL. The corresponding current spectrum and Nyquist plots of the 

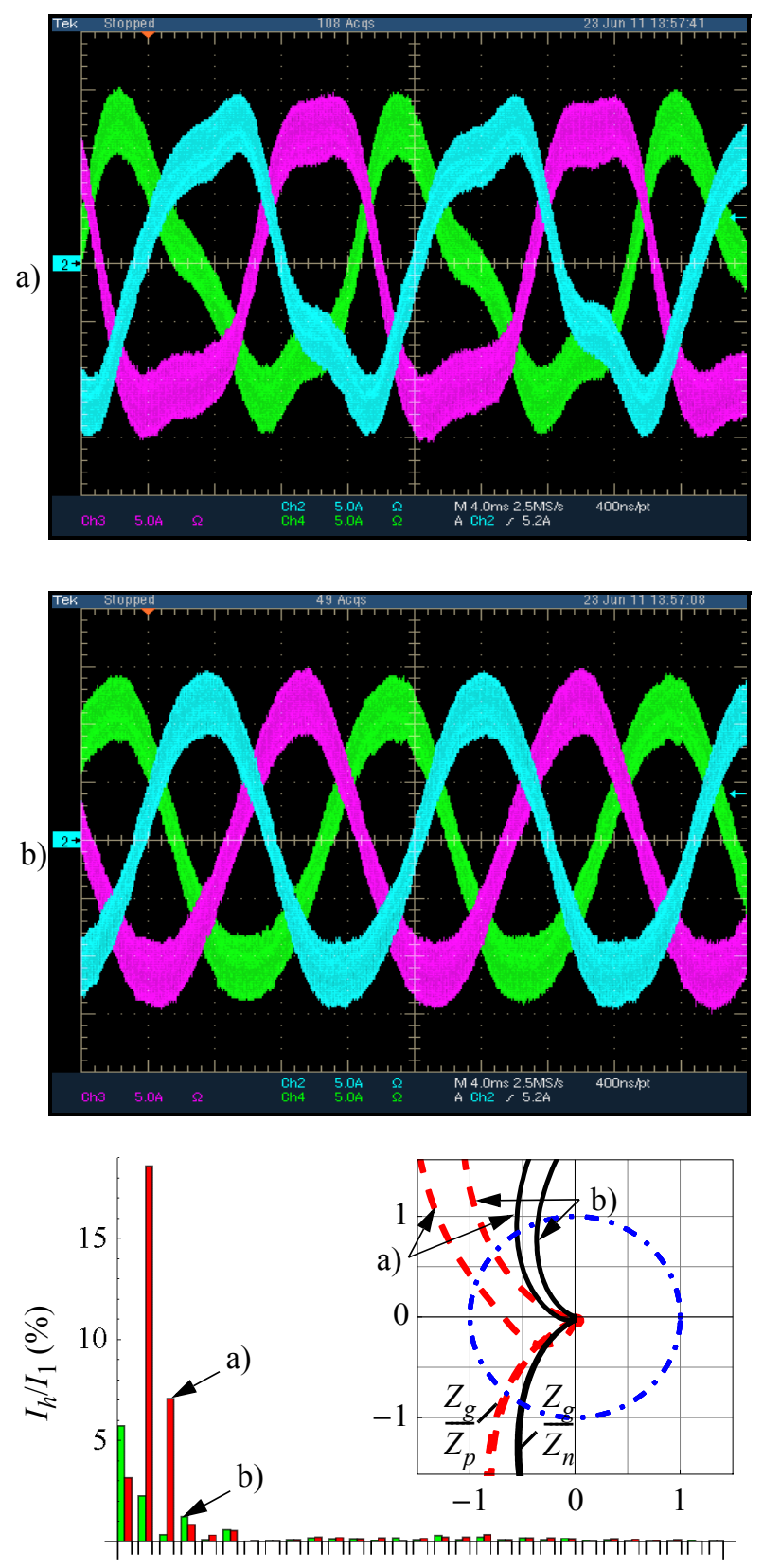

$\begin{array}{llllllllll}3 & 6 & 9 & 12 & 15 & 18 & 21 & 24 & 27 & 30\end{array}$

Harmonic Order

Fig. 12. Mitigation of harmonic resonance in the experimental system by reduction of PLL bandwidth: a) $100 \mathrm{~Hz}$ PLL bandwidth; b) $10 \mathrm{~Hz}$ PLL bandwidth.

impedance ratio are shown in Fig. 12c) as well for comparison with the first case. As can be seen, both the positive- and the negative-sequence impedance ratio has sufficient phase margin in this case, which effectively eliminated the harmonic resonance.

The above examples indicated that slower PLL, although may not track the grid voltage well during transient, tends to improve the compatibility of an inverter with the grid. Other techniques to shape the inverter output impedance to improve grid compatibility include active damping, grid voltage feedforward, and other techniques to shape the inverter output impedance $[16,17]$. Adaptive control of the inverter based on real-time identification of grid impedance is also being developed [18].

\section{Challenges and Future Opportunities}

The discussion so far has focused on grid interface and control of individual inverters. The grid has been considered a passive network that doesn't change often over time. As the level of renewable energy penetration increases, characteristics of the grid, including its impedance, will be more and more dominated by inverters than traditional generators and the network, and the impedance of the grid seen by a particular inverter would be more affected by neighboring inverters than by the actual "grid". In such cases, interactions and possible resonance among neighboring inverters would be more important than that between an inverter and the grid. The impedance-based approach can still be used to characterize such multi-inverter system resonance by applying it to different interface points. However, mitigating resonance and other stability and power quality problems in such multi-converter systems poses some new challenges, particularly when the inverters are manufactured and/or owned by different entities. Adaptive control of an inverter, for example, could interfere with adaptive control of other inverters and create new problems.

Deeper penetration of renewable energy in the distribution network would also increase the frequency and magnitude of local, short-duration voltage fluctuation due to the intermittency of renewable source outputs. Reactive power compensation alone would not be sufficient in mitigating such voltage fluctuation even with fast control, because the problem is largely caused by active power variation and the impedance of the distribution network is mostly resistive. One possible solution is the use of fast-acting energy storage devices such as batteries, and developing advanced control for such distributed voltage regulation devices represents an opportunity for future research.

Electronic devices such as computers, solid-state lighting, variable-speed drives, and battery chargers that are powered from the grid through power electronics interfaces are required to comply with certain electromagnetic interference (EMI) limits. Grid-connected inverters for renewable energy application have to comply with harmonics and other power quality limits [19], but so far there are no EMI limits for such inverters. As the penetration level of renewable energy increases, it will become necessary to limit the EMI emission from gridconnected inverters. The use of EMI filters to comply with such EMI limits will not only increase the size and cost of the inverter, but also add more complexity to the output impedance of the inverter and create more potential for harmonic resonance and other system power quality problems. Eventually, a system approach that considers stability, power quality and EMI is needed to achieve optimal overall performance.

\section{Acknowledgements}

This work was supported in part by the New York State Foundation for Science, Technology and Innovation, the National Science Foundation under Award \#ECCS1002265, the Stanford GCEP, and by GE. 


\section{References}

[1] P. Kundur, Power System Stability and Control, McGrawHill, Inc., 1994.

[2] R. C. Dugan, M. F. McGranaghan, S. Santoso, and H. W. Beaty, Electrical Power Systems Quality, McGraw-Hill, 2nd Ed., 2002.

[3] J. Sun, M. Chen and K. J. Karimi, "Analysis and mitigation of system interactions involving single-phase PFC converters," IEEE Transactions on Aerospace and Electronic Systems, vol. 44, no. 1, pp.217-226, 2008.

[4] Z. Bing and J. Sun, "Line-frequency rectifier dc bus voltage instability analysis and mitigation," in Proceedings of 2010 IEEE Control and Modeling for Power Electronics (COMPEL) Workshop, June 2010.

[5] M. Cespedes, L. Xing, T. Beechner, and J. Sun, "Stabilization of constant-power loads by passive impedance damping," in Proceedings of 2010 IEEE Applied Power Electronics Conference, pp. 2174-2180, February 2010.

[6] L. Xing, F. Feng and J. Sun, "Optimal damping of EMI filter input impedance," IEEE Transactions on Industry Applications, vol. 47, no. 3, pp. 1432-1440, May/June 2011.

[7] M. Liserre, R. Theodorescu and F. Blaabjerg, "Stability of photovoltaic and wind turbine grid-parallel inverters for a large set of grid impedance values," IEEE Transactions on Power Electronics, vol. 21, no. 1, pp. 263-272, 2006.

[8] J. Sun, "Impedance-based stability criterion for gridconnected inverters," IEEE Transactions on Power Electronics, vol. 27, no. 11, Nov. 2011.

[9] J. Sun, "Small-signal methods for ac distributed power systems," IEEE Transactions on Power Electronics, vol. 24, no. 11, pp. 2545-2554, November 2009.

[10] Z. Yao, P.G. Therond and B. Davat, "Stability analysis of power systems by the generalized Nyquist criterion," in Proceedings of International Conference on Control'94, pp. 739-744, March 1994.

[11] J. Sun, Z. Bing, and K. Karimi, "Input impedance modeling of multipulse rectifiers by harmonic linearization," IEEE
Transactions on Power Electronics, vol. 24, no. 12, pp. 2812-2820, December 2009.

[12] Z. Bing, K. Karimi, and J. Sun, "Input impedance modeling and analysis of line-commutated rectifiers," IEEE Transactions on Power Electronics, vol. 24, no. 10, pp. 2338-2346, October 2009.

[13] M. Cespedes and J. Sun, "Renewable energy systems instability involving grid-parallel inverters," in Proceedings of 2009 IEEE Applied Power Electronics Conference, pp. 1971-1977, 2009.

[14] M. Cespedes and J. Sun, "Impedance modeling and control of grid-parallel inverters," in Proceedings of $10^{\text {th }}$ International Workshop on Large-Scale Integration of Wind Power into Power Systems as well as on Transmission Networks for Offshore Wind Power Plans, Oct. 25-26, 2011, Aarhus, Denmark.

[15] H. Liu and J. Sun, "A study of offshore wind HVDC system stability and control," in Proceedings of $10^{\text {th }}$ International Workshop on Large-Scale Integration of Wind Power into Power Systems as well as on Transmission Networks for Offshore Wind Power Plans, Oct. 25-26, 2011, Aarhus, Denmark.

[16] M. Cespedes and J. Sun, "Modeling and mitigation of harmonic resonance between wind turbines and the grid," in Proceedings of IEEE 2011 Energy Conversion Congress and Exposition (ECCE 2011), Phoenix, AZ, September 2011.

[17] M. Cespedes and J. Sun, "Impedance shaping of three-phase grid-parallel voltage-source converters," to presented at IEEE 2012 Applied Power Electronics Conference, February 2012.

[18] M. Cespedes and J. Sun, "Online identification of grid impedance for adaptive control of grid-connected inverters," submitted to IEEE 2012 Energy Conversion Congress and Exposition (ECCE 2012).

[19] IEEE Standard for Interconnecting Distributed Resources with Electric Power Systems, IEEE Standard Coordinating Committee 21, July 2003. 